\title{
Boundary Value Problems with Integral Gluing Conditions for Fractional-Order Mixed-Type Equation
}

\author{
A. S. Berdyshev, ${ }^{1}$ E. T. Karimov, ${ }^{2}$ and N. Akhtaeva ${ }^{1}$ \\ ${ }^{1}$ Kazakh National Pedagogical University named after Abai, 050010 Almaty, Kazakhstan \\ ${ }^{2}$ University of Santiago de Compostela, 15782 Santiago de Compostela, Spain
}

Correspondence should be addressed to E. T. Karimov, erkinjon.karimov@usc.es

Received 18 May 2011; Accepted 11 October 2011

Academic Editor: Shaher M. Momani

Copyright (C) 2011 A. S. Berdyshev et al. This is an open access article distributed under the Creative Commons Attribution License, which permits unrestricted use, distribution, and reproduction in any medium, provided the original work is properly cited.

\begin{abstract}
Analogs of the Tricomi and the Gellerstedt problems with integral gluing conditions for mixed parabolic-hyperbolic equation with parameter have been considered. The considered mixed-type equation consists of fractional diffusion and telegraph equation. The Tricomi problem is equivalently reduced to the second-kind Volterra integral equation, which is uniquely solvable. The uniqueness of the Gellerstedt problem is proven by energy integrals' method and the existence by reducing it to the ordinary differential equations. The method of Green functions and properties of integral-differential operators have been used.
\end{abstract}

\section{Introduction}

Mathematical model of the movement of gas in a channel surrounded by a porous environment was described by parabolic-hyperbolic equation. This was done in the fundamental work of Gel'fand [1]. Modeling of heat transfer processes in composite environment with finite and infinite velocities leads to boundary value problems (BVPs) for parabolic-hyperbolic equations [2]. Omitting the huge amount of works devoted to studying these kinds of equations, we refer the readers to $[3,4]$.

We would like to note works [5-10], devoted to the studying of BVPs for parabolichyperbolic equations, involving fractional derivatives. In turn, applications of Fractionalorder differential equations can be found in the monographs [11-15]. We also note some recent papers [16-18], related to the fractional diffusion and diffusion-wave equations.

BVP for parabolic-hyperbolic equations with integral gluing condition for the first time was investigated by Kapustin and Moiseev [19] and was generalized for this kind of equation, 
but with parameters, in the work [20]. Another motivation of the usage of integral gluing conditions comes from the appearance of them in heat exchange processes [21].

The consideration of equations with parameters was interesting because of the possibility of studying some multidimensional analogues of the main BVP via reducing them by Fourier transformation to the BVP for equations with parameters. On the other hand, consideration of equations with parameters will give possibility to study some spectral properties of BVPs for this kind of equations such as the existence of nontrivial solutions for corresponding homogeneous problem at some values of parameters [22].

\section{Analog of the Tricomi Problem}

Consider an equation

$$
u_{x x}-D_{0 y}^{\alpha H(x)+2 H(-x)} u=\lambda u
$$

in the domain $\Omega=\Omega_{1} \cup A A_{0} \cup \Omega_{2}$. Here $\Omega_{1}=\{(x, y): 0<x<1,0<y<1\}, \Omega_{2}$ is characteristic triangle with endpoints $A(0,0), A_{0}(0,1), C(-1 / 2,1 / 2), H(x)$ is Heaviside function,

$$
D_{a t}^{\alpha} f(t)=\frac{1}{\Gamma(n-\alpha)}\left(\frac{d}{d t}\right)^{n} \int_{a}^{t}(t-s)^{-\alpha+n-1} f(s) d s
$$

is the $\alpha$ th Riemann-Liouville fractional-order derivative of a function $f$ given on interval $[a, b]$, where $n=[\alpha]+1$ and $[\alpha]$ is the integer part of $\alpha$, and $\Gamma(\cdot)$ is the Euler gamma function defined by

$$
\Gamma(\alpha)=\int_{0}^{\infty} t^{\alpha-1} e^{-t} d t, \quad \alpha>0
$$

For $\lambda>0$ and $0<\alpha \leq 1$ given, we formulate the following problem called the analog of the Tricomi problem.

\section{Problem AT}

To find a solution of (2.1), which belongs to the class of functions

$$
W_{1}=\left\{u: D_{0 y}^{\alpha-1} u \in C\left(\overline{\Omega_{1}}\right), u_{x x}, D_{0 y}^{\alpha} u \in C\left(\Omega_{1}\right), u_{x}\left(0^{ \pm}, y\right) \in H(0 ; 1), u \in C\left(\overline{\Omega_{2}}\right) \cap C^{2}\left(\Omega_{2}\right)\right\}
$$

satisfying the initial condition

$$
\lim _{y \rightarrow 0} y^{1-\alpha} u(x, y)=\omega(x), \quad 0 \leq x \leq 1
$$

together with the boundary conditions

$$
\begin{gathered}
u(-y / 2, y / 2)=\psi_{1}(y), \quad 0 \leq y \leq 1, \\
u(1, y)=\psi_{2}(y), \quad 0 \leq y \leq 1,
\end{gathered}
$$


and the gluing conditions

$$
\begin{gathered}
u\left(0^{-}, y\right)=\frac{1}{\Gamma(1-\alpha)} \int_{0}^{y} u\left(0^{+}, t\right)(y-t)^{-\alpha} d t, \quad 0<y \leq 1 \\
\int_{0}^{y} u_{x}\left(0^{-}, t\right) J_{0}[\sqrt{\lambda}(y-t)] d t=\frac{1}{\Gamma(1-\alpha)} \int_{0}^{y} u_{x}\left(0^{+}, t\right)(y-t)^{-\alpha} d t, \quad 0<y<1 .
\end{gathered}
$$

Here $\omega(x), \psi_{i}(y)(i=1,2)$ are given functions such as $\lim _{\mathrm{y} \rightarrow 0} y^{1-\alpha} \psi_{1}(y)=\omega(0)$.

Solution of the Cauchy problem for (2.1) in $\Omega_{2}$ defined as

$$
\begin{gathered}
u(x, y)=\frac{1}{2}\left\{\tau^{-}(y+x)+\tau^{-}(y-x)+\int_{y-x}^{y+x} v^{-}(t) J_{0}\left[\sqrt{\lambda\left[(y-t)^{2}-x^{2}\right]}\right] d t\right. \\
\left.+\lambda x \int_{y-x}^{y+x} \tau^{-}(t) \frac{J_{1}\left[\sqrt{\lambda\left[(y-t)^{2}-x^{2}\right]}\right]}{\sqrt{\lambda\left[(y-t)^{2}-x^{2}\right]}} d t\right\},
\end{gathered}
$$

where $J_{k}[\cdot]$ is the first-kind Bessel function of the order $k, \tau^{-}(y)=u\left(0^{-}, y\right), v^{-}(y)=u_{x}\left(0^{-}, y\right)$. We calculate $u(-y / 2, y / 2)$ in order to use condition (2.5):

$$
\begin{aligned}
& u(-y / 2, y / 2) \\
& \quad=\frac{1}{2}\left\{\tau^{-}(0)+\tau^{-}(y)-\int_{0}^{y} v^{-}(t) J_{0}[\sqrt{\lambda t(t-y)}] d t+\lambda \frac{y}{2} \int_{0}^{y} \tau^{-}(t) \frac{J_{1}[\sqrt{\lambda t(t-y)}]}{\sqrt{\lambda t(t-y)}} d t\right\} .
\end{aligned}
$$

Considering the condition (2.5) and the following integral operator [23]

$$
B_{m x}^{n, \sqrt{\lambda}}[f(x)]=f(x)+\int_{m}^{x} f(t)\left(\frac{x-m}{t-m}\right)^{1-n} \frac{\partial}{\partial x} J_{0}[\sqrt{\lambda(t-m)(t-x)}] d t, \quad m, n=0,1
$$

equality (2.9) can be written as follows

$$
\psi_{1}(y)=\frac{1}{2}\left\{\psi_{1}(0)+B_{0 y}^{0, \sqrt{\lambda}}\left[\tau^{-}(y)\right]-\int_{0}^{y} B_{0 t}^{1, \sqrt{\lambda}}\left[\mathcal{v}^{-}(t)\right] d t\right\}
$$

Now we use an integral operator

$$
A_{m x}^{n, \sqrt{\lambda}}[f(x)]=f(x)-\int_{m}^{x} f(t)\left(\frac{t-m}{x-m}\right)^{n} \frac{\partial}{\partial t} J_{0}[\sqrt{\lambda(x-m)(x-t)}] d t, \quad m, n=0,1,
$$


which is mutually inverse with the operator (2.10). Applying the operator (2.12) to both sides of (2.11), we obtain

$$
A_{0 y}^{0, \sqrt{\lambda}}\left[\psi_{1}(y)\right]=\frac{1}{2}\left\{\psi_{1}(0)+A_{0 y}^{0, \sqrt{\lambda}}\left\{B_{0 y}^{0, \sqrt{\lambda}}\left[\tau^{-}(y)\right]\right\}-A_{0 y}^{0, \sqrt{\lambda}}\left\{\int_{0}^{y} B_{0 t}^{1, \sqrt{\lambda}}\left[v^{-}(t)\right] d t\right\}\right\} .
$$

Considering the following properties of operators (2.10) and (2.12)

$$
A_{0 y}^{0, \sqrt{\lambda}}\left\{B_{0 y}^{0, \sqrt{\lambda}}[f(y)]\right\}=f(y), \quad A_{0 y}^{0, \sqrt{\lambda}}\left\{\int_{0}^{y} B_{0 t}^{1, \sqrt{\lambda}}[f(t)] d t\right\}=\int_{0}^{y} f(t) J_{0}[\sqrt{\lambda}(y-t)] d t
$$

we derive

$$
2 A_{0 y}^{0, \sqrt{\lambda}}\left[\psi_{1}(y)\right]=\psi_{1}(0)+\tau^{-}(y)-\int_{0}^{y} v^{-}(t) J_{0}[\sqrt{\lambda}(y-t)] d t
$$

Taking gluing conditions (2.7) into account, we have

$$
D_{0 y}^{\alpha-1} \mathcal{v}^{+}(y)=D_{0 y}^{\alpha-1} \tau^{+}(y)-2 A_{0 y}^{0, \sqrt{\lambda}}\left[\psi_{1}(y)\right]+\psi_{1}(0)
$$

Applying operator $D_{0 y}^{1-\alpha}$ to both sides of (2.16) and considering the following composition rule [11]:

$$
D_{a t}^{\alpha} D_{a t}^{\beta} f(t)=D_{a t}^{\alpha+\beta} f(t), \quad \beta \leq 0,
$$

we get

$$
\tau^{+}(y)=v^{+}(y)+\psi_{1}^{*}(y), \quad 0<y<1
$$

where $\psi_{1}^{*}(y)=D_{0 y}^{1-\alpha}\left\{2 A_{0 y}^{0, \sqrt{\lambda}}\left[\psi_{1}(y)\right]-\psi_{1}(0)\right\}$.

Let us consider the following auxiliary problem:

$$
\begin{gathered}
u_{x x}-D_{0 y}^{\alpha} u-\lambda u=0, \\
u_{x}(0, y)=v^{+}(y), \quad u(1, y)=\psi_{2}(y), \quad \lim _{y \rightarrow 0} y^{1-\alpha} u(x, y)=\omega(x) .
\end{gathered}
$$

Solution of this problem can be defined as [24]

$$
\begin{aligned}
u(x, y)= & \int_{0}^{1} \omega(\xi) G(x, y, \xi, 0) d \xi-\int_{0}^{y} v^{+}(\eta) G(x, y, 0, \eta) d \eta \\
& +\int_{0}^{y} \psi_{2}(\eta) G_{\xi}(x, y, 1, \eta) d \eta-\lambda \int_{0}^{1} \int_{0}^{y} u(\xi, \eta) G(x, y, \xi, \eta) d \xi d \eta
\end{aligned}
$$


where

$$
G(x, y, \xi, \eta)=\frac{(y-\eta)^{\beta-1}}{2} \sum_{n=-\infty}^{\infty}\left[e_{1, \beta}^{1, \beta}\left(-\frac{|x-\xi+2 n|}{(y-\eta)^{\beta}}\right)+e_{1, \beta}^{1, \beta}\left(-\frac{|x+\xi+2 n|}{(y-\eta)^{\beta}}\right)\right]
$$

is the Green function of the problem (2.19),

$$
e_{1, \beta}^{1, \beta}(z)=\Phi(-\beta, \beta, z)=\sum_{n=0}^{\infty} \frac{z^{n}}{n ! \Gamma(-\beta n+\beta)}
$$

is the function of Wright [25], $\beta=\alpha / 2$.

Considering (2.20) as an integral equation regarding the function $u(x, y)$, we write solution via resolvent of the kernel $\lambda G(x, y, \xi, \eta)$ :

$$
u(x, y)=P(x, y)-\int_{0}^{y} v^{+}(\eta) K_{1}(x, y, \eta) d \eta,
$$

where

$$
\begin{aligned}
P(x, y)= & \int_{0}^{1} \omega(\xi) G(x, y, \xi, 0) d \xi+\int_{0}^{y} \int_{0}^{1} \int_{0}^{1} \omega(\xi) G(s, t, \xi, 0) R(x, y, \xi, 0) d \xi d s d t \\
& +\int_{0}^{y} \psi_{2}(\eta)\left[G(x, y, 1, \eta)+\int_{\eta}^{y} \int_{0}^{1} G(s, t, 1, \eta) R(x, y, 1, \eta) d s d t\right] d \eta, \\
K_{1}(x, y, \eta)= & G(x, y, 0, \eta)+\int_{\eta}^{y} \int_{0}^{1} G(s, t, 0, \eta) R(x, y, 0, \eta) d s d t,
\end{aligned}
$$

$R(x, y, \xi, \eta)$ is a resolvent of the kernel $\lambda G(x, y, \xi, \eta)$.

From (2.23), tending $x$ to $0^{+}$, we obtain

$$
u\left(0^{+}, y\right)=\tau^{+}(y)=P\left(0^{+}, y\right)-\int_{0}^{y} v^{-}(\eta) K_{1}\left(0^{+}, y, \eta\right) d \eta .
$$

Considering functional relation (2.18), from (2.25) we get

$$
v^{+}(y)+\int_{0}^{y} v^{+}(\eta) K_{1}(y, \eta) d \eta=\psi_{1}^{*}(y)-P(0, y)
$$

Equality (2.26) is the second-kind Volterra-type integral equation regarding the function $v^{+}(y)$. Since kernel $K_{1}(y, \eta)$ has weak singularity and functions on the right-hand side are continuous, we can conclude that (2.26) is uniquely solvable [26], and solution can be represented as

$$
v^{+}(y)=\Psi(y)+\int_{0}^{y} \Psi(\eta) K_{2}(y, \eta) d \eta
$$

where $\Psi(y)=\psi_{1}^{*}(y)-P(0, y), K_{2}(y, \eta)$ is the resolvent of the kernel $K_{1}(y, \eta)$. 
Once we have obtained $v^{+}(y)$, considering (2.18) or (2.25) we find function $\tau^{+}(y)$. Then using gluing conditions (2.7) we find functions $\tau^{-}(\mathrm{y}), v^{-}(\mathrm{y})$. Finally, we can define solution of the considered problem by the formula (2.23) in the domain $\Omega_{1}$, by formula (2.8) in the domain $\Omega_{2}$.

Hence, we prove the following theorem.

Theorem 2.1. If

$$
\omega(x) \in C^{2}[0,1], \quad \psi_{i}(y) \in C^{1}[0,1] \cap C^{2}(0,1) \quad(i=1,2),
$$

then there exists unique solution of the Problem AT and is defined by formulas (2.23) and (2.8) in the domains $\Omega_{1}, \Omega_{2}$, respectively.

\section{Analog of the Gellerstedt Problem}

We would like to note some related works. Regarding the consideration of Gellerstedt problem for parabolic-hyperbolic equations with constant coefficients we refer the readers to [3] and for loaded parabolic-hyperbolic equations work by Khubiev [27], and also for Lavrent'ev-Bitsadze equation [28].

Consider an equation

$$
0= \begin{cases}u_{x x}-D_{0 y}^{\alpha} u-\lambda u, & \Phi_{0} \\ u_{x x}-u_{y y}+\lambda u, & \Phi_{i},(i=1,2)\end{cases}
$$

in the domain $\Phi=\left(\bigcup_{k=0}^{2} \Phi_{k}\right) \cup I_{0}$, where $\Phi_{0}$ is a domain, bounded by segments $A A_{0}, B B_{0}, A_{0} B_{0}$ of straight lines $x=0, x=1, y=1$, respectively; $\Phi_{1}$ is a domain, bounded by the segment $\mathrm{AE}$ of the axe $x$ and by characteristics of (3.1) $A C_{1}: x+y=0, E C_{1}: x-y=r ; \Phi_{2}$ is a domain, bounded by the segment $E B$ of the axe $x$ and by characteristics of (3.1) $E C_{2}: x-y=r$, $B C_{2}: x-y=1 ; I_{0}$ is an interval $0<x<1, I_{1}$ is an interval $0<x<r$, and $I_{2}$ is an interval $r<x<1$.

\section{Problem AG}

To find a solution of (3.1) from the class of functions

$$
\begin{aligned}
& W_{2} \\
& =\left\{u: D_{0 y}^{\alpha-1} u \in C\left(\overline{\Phi_{0}}\right), u_{x x}, D_{0 y}^{\alpha} u \in C\left(\Phi_{0}\right), u_{y}\left(x, 0^{ \pm}\right) \in H\left(I_{0}\right), u \in C\left(\overline{\Phi_{i}}\right) \cap C^{2}\left(\Phi_{i}\right)(i=1,2)\right\},
\end{aligned}
$$

satisfying boundary conditions

$$
u(0, y)=\varphi_{1}(y), \quad u(1, y)=\varphi_{2}(y), \quad 0 \leq y \leq 1,
$$


International Journal of Differential Equations

$$
\begin{gathered}
\left.u\right|_{A C_{1}}=u\left(\frac{x}{2},-\frac{x}{2}\right)=\varphi_{3}(x), \quad 0 \leq x \leq r, \\
\left.u\right|_{E C_{2}}=u\left(\frac{(x+r)}{2}, \frac{(r-x)}{2}\right)=\varphi_{4}(x), \quad r \leq x \leq 1,
\end{gathered}
$$

together with gluing conditions

$$
\begin{gathered}
\lim _{y \rightarrow 0^{+}} y^{1-\alpha} u(x, y)=\lim _{y \rightarrow 0^{+}} u(x, y), \quad x \in \overline{I_{0}} \\
\lim _{y \rightarrow 0^{+}}\left[y^{1-\alpha}\left(y^{1-\alpha} u(x, y)\right)_{y}\right]=\int_{0}^{x} \lim _{y \rightarrow 0^{+}} u_{y}(t, y) J_{0}[\sqrt{\alpha}(x-t)] d t, \quad x \in I_{0} \backslash\{\mathrm{r}\} .
\end{gathered}
$$

Here $\varphi_{j}(\cdot)(j=\overline{1,4})$ are given functions such as $\lim _{y \rightarrow 0^{+}} y^{1-\alpha} \varphi_{1}(y)=\varphi_{3}(0), \lim _{\mathrm{y} \rightarrow 0^{+}} y^{1-\alpha} u(r, y)=$ $\varphi_{4}(r)$.

Theorem 3.1. If the following conditions

$$
\lambda \geq 0, \quad \varphi_{i}(y) \in C^{1}[0,1] \cap C^{2}(0,1), \quad \varphi_{j}(x) \in C^{1}\left(\overline{I_{i}}\right) \cap C^{2}\left(I_{i}\right) \quad(i=1,2 ; j=3,4)
$$

are fulfilled, then the Problem AG has a unique solution.

Proof. Introduce the following designations:

$$
\begin{gathered}
\lim _{y \rightarrow 0^{+}} y^{1-\alpha} u(x, y)=\tau^{+}(x), \quad \lim _{y \rightarrow 0^{-}} u(x, y)=\tau^{-}(x), \quad x \in \overline{I_{0}}, \\
\lim _{y \rightarrow 0^{+}}\left[y^{1-\alpha}\left(y^{1-\alpha} u(x, y)\right)_{y}\right]=v^{+}(x), \quad \lim _{y \rightarrow 0^{-}} u_{y}(x, y)=v^{-}(x), \quad x \in I_{0} .
\end{gathered}
$$

Solution of the Cauchy problem for (3.1) in the domain $\Phi_{i}(i=1,2)$ in case, when $\lambda \geq 0$ has a form

$$
\begin{aligned}
& u(x, y)=\frac{1}{2}\left\{\tau^{-}(x+y)+\tau^{-}(x-y)+\int_{x-y}^{x+y} v^{-}(t) J_{0}\left[\sqrt{\lambda\left[(x-t)^{2}-y^{2}\right]}\right] d t\right. \\
& \left.+\lambda y \int_{x-y}^{x+y} \tau^{-}(t) \frac{J_{1}\left[\sqrt{\lambda\left[(x-t)^{2}-y^{2}\right]}\right]}{\sqrt{\lambda\left[(x-t)^{2}-y^{2}\right]}} d t\right\} .
\end{aligned}
$$


Using boundary conditions (3.4), (3.5), and gluing conditions (3.6), (3.7), from (3.10) we obtain

$$
\begin{array}{ll}
v^{+}(x)=\tau^{+}(x)+\varphi_{3}^{*}(x), & x \in I_{1}, \\
v^{+}(x)=\tau^{+}(x)+\varphi_{4}^{*}(x), & x \in I_{2},
\end{array}
$$

where

$$
\varphi_{3}^{*}(x)=\varphi_{3}(0)-A_{0 x}^{0, \sqrt{\lambda}}\left[2 \varphi_{3}(x)\right], \quad \varphi_{4}^{*}(x)=\varphi_{4}(r)-A_{r x}^{0, \sqrt{\lambda}}\left[2 \varphi_{4}(x)\right] .
$$

According to [10], tending $y$ to +0 , from (3.1) we get

$$
v^{+}(y)=\frac{1}{\Gamma(1+\alpha)}\left[\tau^{+\prime \prime}(x)-\lambda \tau^{+}(x)\right]
$$

In order to prove the uniqueness of the solution for the Problem AG, we need estimate the following integral:

$$
\mathbb{I}=\int_{0}^{1} \tau^{+}(x) v^{+}(x) d x
$$

Considering homogeneous case of the condition (3.3) and taking designation (3.9) into account, after some evaluations we derive

$$
\mathbb{I}=-\int_{0}^{1}\left\{\left[\tau^{+\prime}(x)\right]^{2}+\lambda\left[\tau^{+}(x)\right]^{2}\right\} d x
$$

If $\lambda \geq 0$, then $\mathbb{I} \leq 0$. On the other hand, if we consider homogeneous cases of (3.11) and (3.12), one can easily be sure that $\mathbb{I} \geq 0$. Hence, we get that $\mathbb{I} \equiv 0$. Based on (3.16) we can conclude that $\tau^{+}(x)=0$ for all $x \in \overline{I_{0}}$. Due to the solution of the first boundary problem [24] we can conclude that $u(x, y) \equiv 0$ in $\overline{\Phi_{0}}$. Further, according to the gluing conditions and the solution of Cauchy problem, we have $u(x, y) \equiv 0$ in $\bar{\Phi}$.

Considering functional relations (3.11)-(3.14) and conditions (3.3)-(3.5), we get the following problems:

$$
\begin{gathered}
\tau^{+\prime \prime}(x)-(\lambda+\Gamma(1+\alpha)) \tau^{+}(x)=\varphi_{3}^{*}(x) \Gamma(1+\alpha), \\
\tau^{+}(0)=\varphi_{3}(0), \quad \tau^{+}(r)=\varphi_{4}(r), \quad x \in \overline{I_{1}}, \\
\tau^{+\prime \prime}(x)-(\lambda+\Gamma(1+\alpha)) \tau^{+}(x)=\varphi_{4}^{*}(x) \Gamma(1+\alpha), \\
\tau^{+}(r)=\varphi_{4}(r), \quad \tau^{+}(1)=\lim _{y \rightarrow+0} y^{1-\alpha} \varphi_{2}(y), \quad x \in \overline{I_{1}} .
\end{gathered}
$$


The problems (3.17) and (3.18) are model problems and can be solved directly. After the finding function $\tau^{+}(x)$ for all $x \in \overline{I_{0}}$, functions $v^{+}(x)$ and $\tau^{-}(x), v^{-}(x)$ can be defined by formulas (3.14) and (3.6), (3.7), respectively. Finally, solution of the Problem AG can be recovered by formulas (3.10) and (2.23) in the domains $\Phi_{i}(i=1,2)$ and $\Phi_{0}$, respectively, but only with some changes in (2.23), precisely, Green function $G(x, y, \xi, \eta)$ should be replaced by

$$
G^{*}(x, y, \xi, \eta)=\frac{(y-\eta)^{\beta-1}}{2} \sum_{n=-\infty}^{\infty}\left[e_{1, \beta}^{1, \beta}\left(-\frac{|x-\xi+2 n|}{(y-\eta)^{\beta}}\right)-e_{1, \beta}^{1, \beta}\left(-\frac{|x+\xi+2 n|}{(y-\eta)^{\beta}}\right)\right]
$$

which is the Green function of the first boundary problem for the (3.1) in $\Phi_{0}$ [24].

Theorem 3.1 is proved.

\section{Acknowledgment}

This paper is partially supported by the foundation ERASMUSMUNDUS (Project number 155778-EM-1-2009-1-BEERASMUNMUNDUS-ECW-LO9).

\section{References}

[1] I. M. Gel'fand, "Some questions of analysis and differential equations," Uspekhi Matematicheskikh Nauk, vol. 3, no. 87, pp. 3-19, 1959 (Russian).

[2] V. A. Nakhusheva, Differential Equations of Mathematical Models of Non-Local Processes, Nauka, Moscow, Russia, 2006 (Russian).

[3] T. D. Djuraev, A. Sopuev, M. Mamajonov et al., Boundary Value Problems for Parabolic-Hyperbolic Type Equations, Fan, Tashkent, Uzbekistan, 1986 (Russian).

[4] A. M. Nakhushev, Problems with Displacements for Partial Differential Equations, Nauka, Moscow, Russia, 2006 (Russian).

[5] A. A. Kilbas and O. A. Repin, "An analog of the Tricomi problem for a mixed type equation with a partial fractional derivative," Fractional Calculus E Applied Analysis, vol. 13, no. 1, pp. 69-84, 2010.

[6] V. A. Nakhusheva, "Boundary problems for mixed type heat equation," Doklady AMAN, vol. 12, no. 2, pp. 39-44, 2010 (Russian).

[7] E. Y. Arlanova, "A problem with a shift for the mixed type equation with the generalized operators of fractional integration and differentiation in a boundary condition," Vestnik Samarsk Gosudarstvennogo Universiteta, vol. 6, no. 65, pp. 396-406, 2008 (Russian).

[8] A. A. Kilbas and O. A. Repin, "An analogue of the Bitsadze-Samarskir problem for an equation of mixed type with a fractional derivative," Differentsial'nye Uravneniya, vol. 39, no. 5, pp. 638-719, 2003, Translation in Journal of Difference Equations and Applications, vol. 39, no. 5, pp. 674-680, 2003.

[9] A. V. Efimov, "On boundary value problems with Saigo operators for the mixed type equation with a fractional derivative," Vestnik Samarsk, Georgian Technical University, vol. 26, pp. 16-20, 2004 (Russian).

[10] S. K. Gekkieva, "A boundary value problem for the generalized transfer equation with a fractional derivative in a semi-infinite domain," Izvestiya Kabardino-Balkarskaya Nauchnoogo Tsentra RAN, vol. 8, no. 1, pp. 6-8, 2002 (Russian).

[11] S. G. Samko, A. A. Kilbas, and O. I. Marichev, Fractional Integrals and Derivatives, Gordon and Breach Science, Yverdon, Switzerland, 1993, Edited and with a foreword by S. M. Nikol'ski1. Translated from the 1987 Russian original.

[12] I. Podlubny, Fractional Differential Equations, vol. 198, Academic Press, San Diego, CA, USA, 1999.

[13] A. M. Nakhushev, Fractional Calculus and its Applications, Fizmatlit, Moscow, Russia, 2003.

[14] A. V. Pskhu, Partial Differential Equations of Fractional Order, Nauka, Moscow, Russia, 2005.

[15] A. A. Kilbas, H. M. Srivastava, and J. J. Trujillo, Theory and Applications of Fractional Differential Equations, vol. 204, Elsevier, Amsterdam, The Netherlands, 2006. 
[16] R. Gorenflo and F. Mainardi, "Some recent advances in theory and simulation of fractional diffusion processes," Journal of Computational and Applied Mathematics, vol. 229, no. 2, pp. 400-415, 2009.

[17] Y. Luchko, "Initial-boundary problems for the generalized multi-term time-fractional diffusion equation," Journal of Mathematical Analysis and Applications, vol. 374, no. 2, pp. 538-548, 2011.

[18] W. Chen, H. Sun, X. Zhang, and D. Korošak, "Anomalous diffusion modeling by fractal and fractional derivatives," Computers $\mathcal{E}$ Mathematics with Applications, vol. 59, no. 5, pp. 1754-1758, 2010.

[19] N. Yu. Kapustin and E. I. Moiseev, "On spectral problems with a spectral parameter in the boundary condition," Differentsial'nye Uravneniya, vol. 33, no. 1, pp. 115-143, 1997, Translation in Differential Equations, vol. 33, no. 1, pp. 116-120, 1997.

[20] B. E. Eshmatov and E. T. Karimov, "Boundary value problems with continuous and special gluing conditions for parabolic-hyperbolic type equations," Central European Journal of Mathematics, vol. 5, no. 4, pp. 741-750, 2007.

[21] A. G. Shashkov, System-Structural Analysis of the Heat Exchange Processes and Its Application, Moscow, Russia, 1983.

[22] E. T. Karimov, "Some non-local problems for the parabolic-hyperbolic type equation with complex spectral parameter," Mathematische Nachrichten, vol. 281, no. 7, pp. 959-970, 2008.

[23] M. S. Salakhitdinov and A. K. Urinov, Boundary Value Problems for Equations of Mixed Type with a Spectral Parameter, Fan, Tashkent, Uzbekistan, 1997.

[24] A. V. Pskhu, "Solution of boundary value problems for a diffusion equation of fractional order by the Green's function method," Differentsial'nye Uravneniya, vol. 39, no. 10, pp. 1430-1440, 2003.

[25] E. M. Wright, "The generalized Bessel function of order greater than one," The Quarterly Journal of Mathematics, vol. 11, pp. 36-48, 1940.

[26] M. L. Krasnov, Integral Equations: Introduction to the Theory, Nauka, Moscow, Russia, 1975, Selected topics in higher mathematics for engineers and technological university students.

[27] K. U. Khubiev, "Gellerstedt problem loaded mixed type equation with data on non-parallel characteristics," Manual Templating Model, vol. 3, pp. 187-188, 2007 (Russian).

[28] E. I. Moiseev and N. O. Taranov, "Solution of a Gellerstedt problem for the Lavrent'ev-Bitsadze equation," Differentsial'nye Uravneniya, vol. 45, no. 4, pp. 558-563, 2009. 


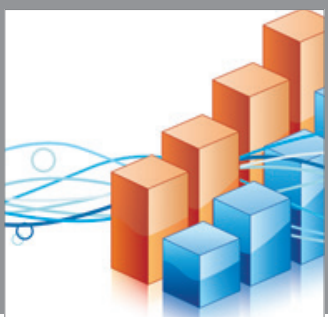

Advances in

Operations Research

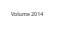

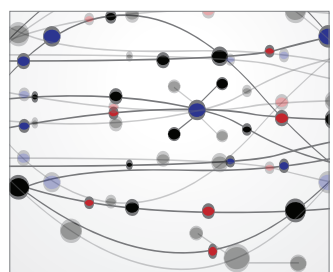

\section{The Scientific} World Journal
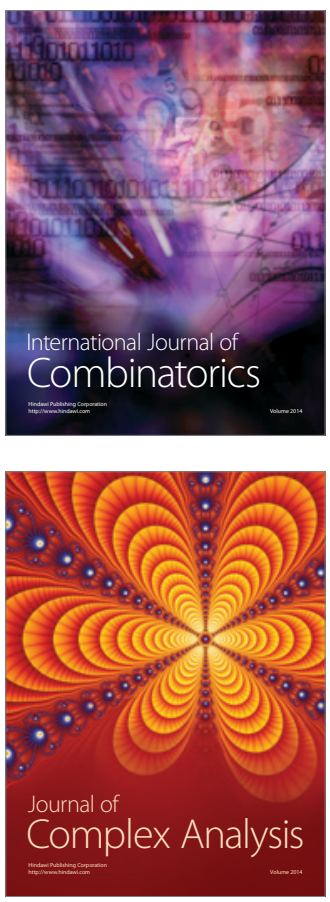

International Journal of

Mathematics and

Mathematical

Sciences
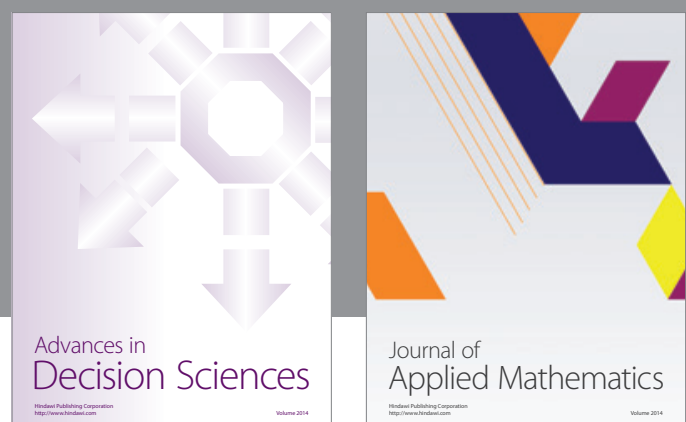

Journal of

Applied Mathematics
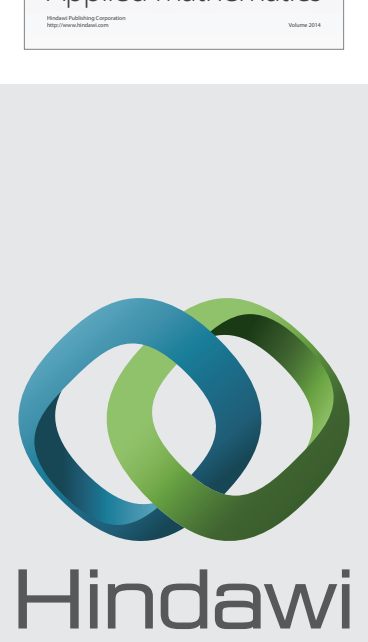

Submit your manuscripts at http://www.hindawi.com
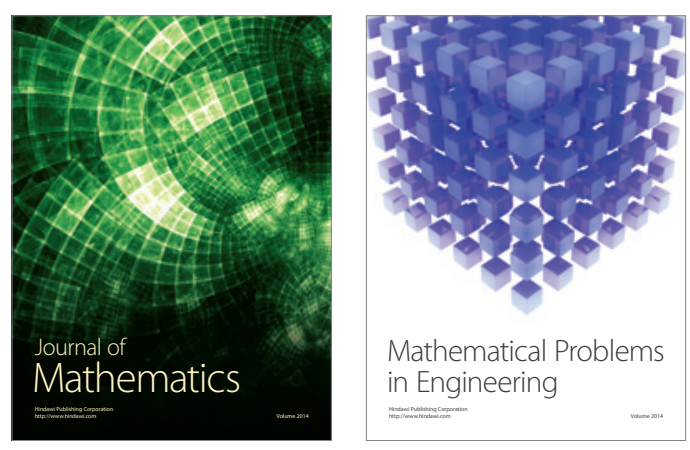

Mathematical Problems in Engineering
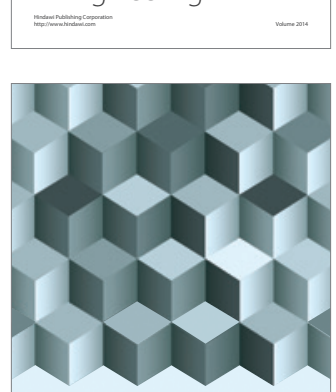

Journal of

Function Spaces
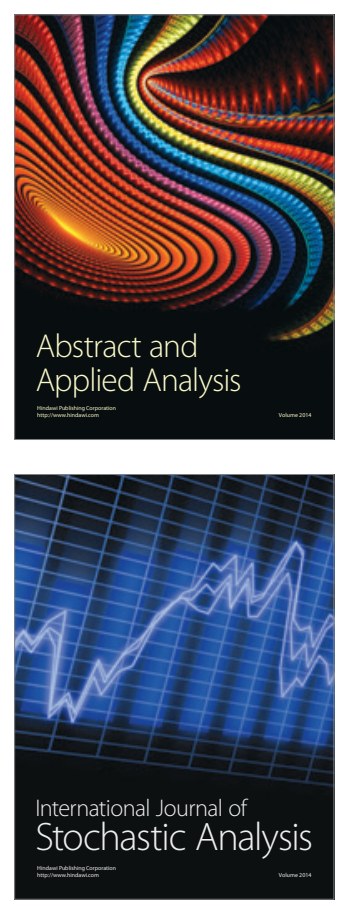

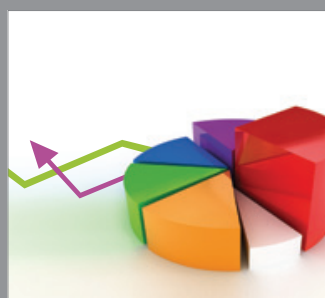

ournal of

Probability and Statistics

Promensencen
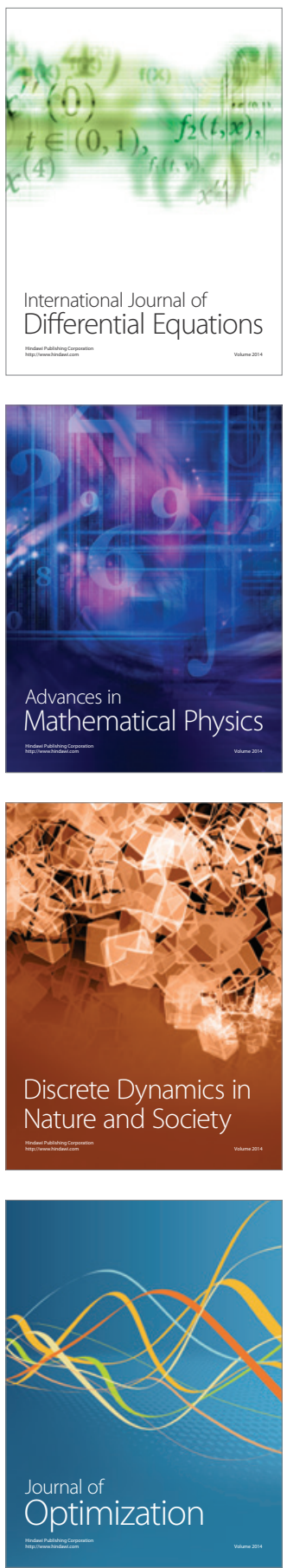